\title{
Nanoscale engineering for sustainable catalysis
}

\author{
The understanding of catalytic nanomaterials and processes results in improved control over their performance to \\ achieve commercial relevance.
}

N anostructured materials are already being widely used in industrial catalytic processes. Advances in synthetic and characterization approaches have facilitated a better understanding of how catalysts work. This in turn guides their rational design and synthesis, resulting in enhanced performances with the goal to achieve the level of industrial relevance, qualified for scale-up pilot studies and eventual commercialization. This issue of Nature Nanotechnology includes a Focus on nanocatalysts for industrial applications that overviews the ways in which catalysts can be engineered at the sub-nano or atomic scale to achieve high selectivity and stability. In particular, we illustrate two examples: nanocatalysts for low-temperature $\mathrm{CO}_{2}$ electrolysis and Pt-based nanostructured catalysts for oxygen reduction reactions in fuel cell vehicles.

Commercial heterogeneous catalysts are usually composed of active sites stabilized on high-surface-area supports. Their performance is determined by various factors including the physical chemistry of the support, and the electronic characteristics, size and morphology of the catalyst. These variables play out in a complex manner and it is difficult to decouple their individual contributions to gain molecular-level understanding. In a Review Article in this issue, Mitchell et al. focus on the surface modifications at the sub-nano or atomic scale, showing how distinct approaches to nanostructural engineering can enhance the selectivity and stability of catalysts for thermo- and electrochemical applications. The advance of aberration-corrected transmission electron microscopy and electron energy-loss spectroscopy enables the identification of isolated single atoms and their coordination environment. The use of simplified models facilitates a better fundamental understanding of structureperformance relationships. However, it remains challenging to completely understand the link between the catalytic reaction dynamics and the geometric and electronic features of the active surface ensembles, which is the key to designing increasingly sophisticated structures with ever more complex behaviours.

Driven by the falling price of renewable energy technologies and the push to decarbonize the economy, the electrocatalytic conversion of $\mathrm{CO}_{2}$ to useful chemicals via electrolysis is growing rapidly. $\mathrm{CO}_{2}$ conversion processes are moving from a laboratory curiosity to a commercially viable process. In a Perspective in this issue, Masel et al. describe the key advances in nanocatalysts that have led to impressive electrochemical conversion of $\mathrm{CO}_{2}$ to useful products and provide benchmarks that one can compare their results to. We learn that the pilot units for the production of $\mathrm{CO}$ from $\mathrm{CO}_{2}$ are now under testing and the pilot study of formic acid production is projected and coming in the next year. The industrially relevant performance of nanocatalysts for $\mathrm{CO}_{2}$ conversion into ethanol and ethylene is also emerging in laboratories. Here, besides catalyst design, innovative reactors and process schemes are also decisive factors that will determine their industrial adoption.

Nanostructured catalysts are engineered to tackle grand challenges such as the transition from fossil to renewable energy resources. Green hydrogen energy, including both hydrogen production from water splitting and its use in fuel cells, has progressed fast during the past decades. For instance, in the article from King et al., the authors demonstrate the translation of a nanoparticulate CoP-based hydrogen catalyst in a commercial polymer electrolyte membrane electrolyser to meet the need to reduce reliance on the scarce and expensive platinum group catalysts ${ }^{1}$, which is important for the large-scale deployment of water electrolysis.

To make hydrogen fuel cells more affordable, catalysts with less Pt and beyond Pt such as precious-metal-free, carbon-based and single-atom catalysts have been widely explored to replace the commercial $\mathrm{Pt} / \mathrm{C}$ catalysts for the oxygen reduction reaction and in laboratories some do exhibit higher activities.
However, their practical applications, for instance in vehicles, are still limited by their low power density and durability. In their Review Article in this issue, Kodama et al. take low Pt loading nanocatalysts as an example, summarizing recent analytical studies to identify the origin of the technical issues, proposing promising strategies for overcoming the trade-offs between the efficiency, power density and durability of polymer electrolyte fuel cells.

The advances in nanostructured catalysts and catalytic processes are not only enabling sustainable energy but also sustainable agriculture. The Haber-Bosch process, which converts hydrogen and nitrogen to ammonia, is used to produce fertilizer and almost all nitrogen-containing commodity chemicals, and is considered one of the most important industrial chemical reactions ever developed. However, the process demands high pressure, high temperature and hydrogen, consuming $1-2 \%$ of the world's energy supply ${ }^{2}$. The electrochemical synthesis of ammonia from nitrogen is a very attractive alternative, due to the mild operation conditions and the use of renewable electricity. In the Article from Han et al., an easy to be scaled-up mechanochemical method was also employed to synthesize ammonia under ambient conditions, with performances rivalling state-of-the-art Haber-Bosch ammonia synthesis ${ }^{3}$. However, there is a long way to go to completely revolutionize the ammonia-manufacturing industry. And for all innovative solutions, accurate sustainability benefits should be quantified to guide further research and aid policymaking.

Published online: 10 February 2021 https://doi.org/10.1038/s41565-021-00862-y

\section{References}

1. King, L. A. et al. Nat. Nanotechnol. 14, 1071-1074 (2019)

2. Kyriakou, V., Garagounis, I., Vourros, A., Vasileiou, E. \& Stoukides, M. Joule 4, 142-158 (2020).

3. Han, G.-F. et al. Nat. Nanotechnol. https://doi.org/10.1038/ s41565-020-00809-9 (2020). 